\title{
Comparison Between the SFTS-QS Kit and the PowerChek SFTSV Real-time PCR Kit for the Detection of Severe Fever With Thrombocytopenia Syndrome Virus
}

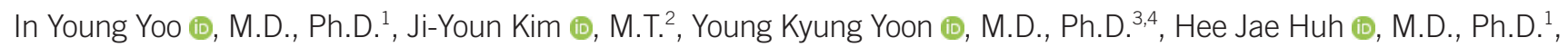 \\ and Nam Yong Lee (1), M.D., Ph.D. ${ }^{1}$ \\ ${ }^{1}$ Department of Laboratory Medicine and Genetics, Samsung Medical Center, Sungkyunkwan University School of Medicine, Seoul, Korea; ${ }^{2} \mathrm{Center}$ for \\ Clinical Medicine, Samsung Biomedical Research Institute, Samsung Medical Center, Seoul, Korea; ${ }^{3}$ Division of Infectious Diseases, Department of Internal \\ Medicine, Korea University College of Medicine, Seoul, Korea; ${ }^{4}$ Institute of Emerging Infectious Diseases, Korea University College of Medicine, Seoul, Korea
}

The recent increase in severe fever with thrombocytopenia syndrome (SFTS) cases has led to the development of the SFTS-QS kit (MiCoBioMed, Seongnam, Korea) for detecting the SFTS virus (SFTSV, now renamed Huaiyangshan banyangviruS). SFTS-QS is a qualitative real-time reverse transcription PCR assay based on lab-on-a- chip technology. We evaluated the performance of the SFTS-QS kit and compared it with that of the PowerChek SFTSV Real-time PCR kit (PowerChek; Kogene Biotech, Seoul, Korea). A total of 117 serum samples were simultaneously assayed using the SFTS-QS and PowerChek kits. Sanger sequencing targeting the $S$ and $M$ segments of SFTSV was performed as the reference method. The total turnaround time of the two kits was compared. The SFTS-QS results agreed with those of PowerChek with a kappa value of 0.92. The diagnostic sensitivity and specificity of the SFTS-QS kit were both $100 \%$ (14/14 and 103/103, respectively), whereas those of the PowerChek kit were 100\% (14/14) and 98.1\% (101/103), respectively. The results of SFTS-QS and PowerChek were comparable; however, the SFTS-QS kit required a shorter total turnaround time. The SFTS-QS kit produced accurate and fast results and thus could serve as a useful tool for detecting SFTSV.

Key Words: Huaiyangshan banyangvirus, SFTS virus, Performance, Turnaround time, SFTSQS kit, PowerChek SFTSV Real-time PCR kit
Received: September 6, 2019

Revision received: November 17, 2019

Accepted: January 15, 2020

Corresponding author: Hee Jae Huh, M.D. Department of Laboratory Medicine and Genetics, Samsung Medical Center, Sungkyunkwan University School of Medicine, 81 Irwon-ro, Gangnam-gu, Seoul 06351, Korea

Tel: +82-2-3410-1836

Fax: +82-2-3410-2719.

E-mail: pmhhj77@gmail.com

\section{(c) (i) (5)}

(c) Korean Society for Laboratory Medicine

This is an Open Access article distributed under the terms of the Creative Commons Attribution Non-Commercial License (https://creativecommons.org/licenses/by-nc/4.0) which permits unrestricted non-commercial use, distribution, and reproduction in any medium, provided the original work is properly cited.
Severe fever with thrombocytopenia syndrome (SFTS) is an emerging hemorrhagic infectious disease caused by the SFTS virus (SFTSV), renamed Huaiyangshan banyangvirus (genus Banyangvirus; family Phenuiviridae) [1]. SFTS has become an endemic disease in central and eastern China, Japan, and Korea, with a high mortality rate $(6.3 \%-30 \%)$ [2, 3]. The clinical manifestation of early stage SFTS is non-specific with fever, headache, myalgia, and gastrointestinal symptoms, followed by thrombocytopenia and leukopenia [4]. In fatal cases, multiple organ failure develops with other clinical symptoms, including disseminated intravascular coagulation, neuropsychiatric symptoms, and sustained thrombocytopenia [2].

Early diagnosis and effective management of SFTSV infections require accurate and reliable diagnostic assays. Several molecular assays have been developed and are currently used for SFTS diagnosis, such as real-time reverse transcription (RT)PCR and loop-mediated isothermal amplification combined with reverse transcription. The majority of molecular assays were de- 
signed to target the L, M, or S segments of the SFTSV genome; of these, the $S$ segment is known to be the most conserved gene [5-10].

The SFTS-QS kit (MiCoBioMed, Seongnam, Korea) is a new molecular assay for the rapid detection of the S segment of SFTSV; it is a one-step real-time RT-PCR kit based on lab-on-a-chip technology. The PowerChek SFTSV Real-time PCR kit (PowerChek kit; Kogene Biotech, Seoul, Korea) is another new one-step realtime RT-PCR kit for the qualitative detection of the $\mathrm{S}$ and $\mathrm{M}$ segments of SFTSV. The performances of the two kits have not been compared previously. We evaluated the analytical and clinical performance of the SFTS-QS kit in comparison with that of the PowerChek kit for the qualitative detection of SFTSV.

This study was approved by the Institutional Review Board (IRB) of the Samsung Medical Center, Seoul, Korea (IRB No. SMC 2016-05-043), and informed consent requirements were waived. From July 2013 to July 2019, a total of 117 serum samples from patients with clinically suspected SFTS were collected and stored at $-70^{\circ} \mathrm{C}$ until analyzed. Eleven other viral pathogens ((Dengue virus type 1 [Hawaii strain, ZeptoMetrix, Buffalo, NY]), Dengue virus type 2 [New Guinea C strain, ZeptoMetrix], Dengue virus type 3 [H87 strain, ZeptoMetrix], Dengue virus type 4 [H241 strain, ZeptoMetrix], Yellow fever virus [17 D strain, ZeptoMetrix], Japanese encephalitis virus [KUMC-27, Korean Bank for Pathogenic Viruses, Seoul, Korea], Seoul virus [clinical isolate], Hantaan virus [clinical isolate], and Zika virus [MR766, PRVABC59 strains, American Type Culture Collection, Rockville, MD; DakArD41662 strain, ZeptoMetrix]) were used to evaluate analytical specificity.

Viral RNA was extracted from the collected serum samples using the QIAamp DSP Viral RNA Mini Kit (QIAamp kit; Qiagen, Hilden, Germany), according to the manufacturer's protocol. The total volume of samples was $200 \mu \mathrm{L}$. The RNA was eluted in $100 \mu \mathrm{L}$, and the two real-time RT-PCR assays were simultaneously performed. The remaining RNA samples were stored at $-70^{\circ} \mathrm{C}$ until use in Sanger sequencing.

Analysis using the SFTS-QS kit was performed on the Veri-Q PCR 316 system (MiCoBioMed, Seongnam, Korea), according to the manufacturer's instructions; the total reaction volume was $10 \mu \mathrm{L}$ (7 $\mu \mathrm{L}$ of PCR mixture and $3 \mu \mathrm{L}$ of template RNA). The SFTSV cut-off value was set at a cycle threshold (Ct) value $\leq 40$. SFTSV-positive/negative controls and an internal control were included in each run.

Analysis using the PowerChek kit was performed on the BioRad CFX96 system (Bio-Rad Laboratories Inc., Hercules, CA, USA) in a total volume of $20 \mu \mathrm{L}(15 \mu \mathrm{L}$ of PCR mixture and $5 \mu \mathrm{L}$ of template RNA), according to the manufacturer's instructions. Positive results were defined by a Ct value $\leq 35$ for the $\mathrm{S}$ or $\mathrm{M}$ segments.

Sanger sequencing targeting the $\mathrm{S}$ and $\mathrm{M}$ segments of SFTSV was used as a reference method and performed according to previous studies [11, 12]. Nested RT-PCR was performed for the $\mathrm{S}$ segment; a second round of amplification was conducted if no product was visible in agarose gel electrophoresis after the first round. Following detection by agarose gel electrophoresis, the PCR product was purified and sequenced.

The analytical specificity of the SFTS-QS kit was determined using the 11 viruses listed above. The limit of detection was evaluated using SFTSV RNA transcripts. Probit analysis was used to determine the 95\% limit of detection, and serial dilutions were analyzed with eight replicates per dilution, according to the Clinical Laboratory Standards Institute (CLSI) EP17-A2 [13]. Precision, including reproducibility and lot-to-lot precision, was determined using two concentrations, according to a modified CLSI EP15-A3 protocol [14]. Reproducibility was assessed by triplicate analysis of each concentration for five days at two different test sites. Lot-to-lot precision was evaluated by analyzing the duplicates of each concentration for five days using two different lots.

The positive percent agreement (PPA) and negative percent agreement (NPA) values and kappa coefficient were calculated to determine the agreement between the results of the SFTS-QS and PowerChek kits. The clinical sensitivities and specificities were calculated based on the results of the reference method. Statistical analyses were performed using the VassarStats website (http://vassarstats.net/).

The total turnaround time, defined as the time interval between reagent preparation for amplification and obtaining the final result, of the SFTS-QS and PowerChek kits was measured for workflow analysis. It was measured for processing five samples in a batch.

Of the 117 serum samples, 14 and 16 were SFTSV-positive by the SFTS-QS and PowerChek kits, respectively; 14 of these samples were identical. The SFTS-QS results were concordant with those of PowerChek for 115/117 (98.3\%) samples. The agreement, including PPAs, NPAs, and kappa values, between the two kits and the accuracy, including sensitivities and specificities, are shown in Table 1. Fourteen samples were SFTSVpositive based on the reference method. The SFTS-QS and PowerChek kits correctly identified 103 and 101 of the 103 SFTSVnegative samples, respectively (Table 1 ). The two samples with false-positive results in the analysis with PowerChek also gave 
Table 1. Performance of the real-time RT-PCR kits for SFTSV detection

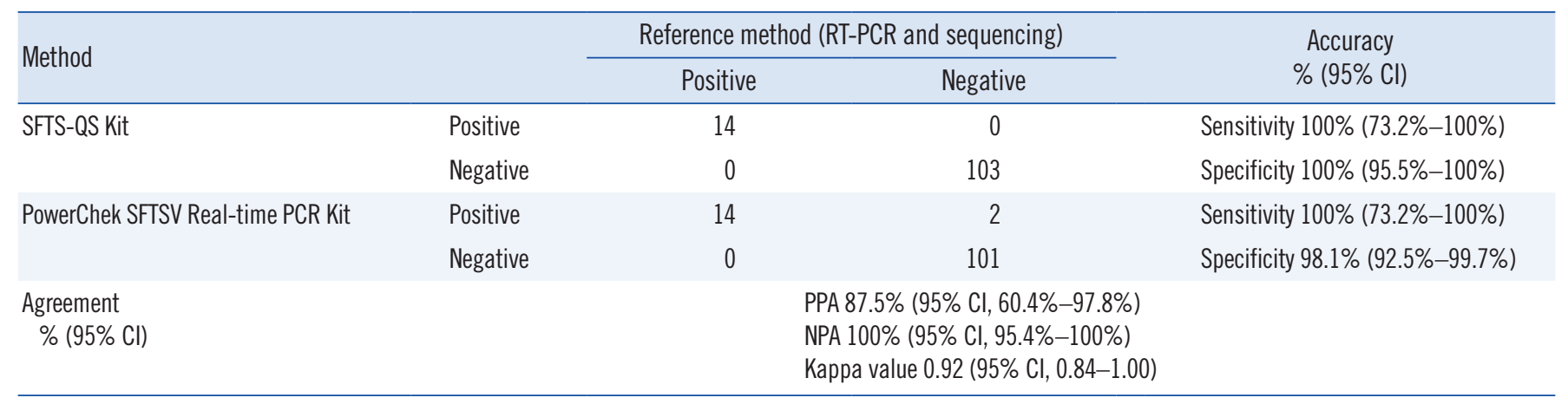

Abbreviations: RT-PCR, reverse transcription PCR; SFTSV, severe fever with thrombocytopenia syndrome virus; Cl, confidence interval, PPA, positive percent agreement, NPV negative percent agreement.

negative results in an assay conducted by the Korea Centers for Disease Control \& Prevention.

The 95\% limit of detection of the SFTS-QS kit was 5.3 copies/ $\mu \mathrm{L}$. In the analytical specificity assay, all 11 viruses gave negative results and no cross-reactivity was observed. In the reproducibility assay, the coefficient of variation (CV) ranged from $2.46 \%$ to $3.65 \%$. The lot-to-lot variation CV ranged from 3.13\% to $3.49 \%$. The total time (55 minutes) required to perform the SFTS-QS assay was shorter than that required for the PowerChek assay (135 minutes).

The recent increase in SFTS cases in East Asia has highlighted the need for a reliable and rapid diagnostic method [2-4]. Sun, et al. [9] developed a quantitative real-time RT-PCR assay targeting the $\mathrm{L}, \mathrm{M}$, and $\mathrm{S}$ genes of SFTSV, which showed $98.6 \%$ clinical diagnostic sensitivity and over $99.0 \%$ specificity. In addition, a two-tube multiplex real-time RT-PCR assay developed by Yoshikawa, et al. [10] for SFTSV detections exhibited a sensitivity of $100 \%$ and a specificity of over $99 \%$. Our study demonstrated that the SFTS-QS and PowerChek kits have good sensitivity and specificity for SFTS diagnosis.

The SFTS-QS assay performed on the Veri-Q PCR 316 system required shorter total turnaround time compared with the PowerChek kit. Based on microfluidic devices (also known as labon-a-chip devices), the Veri-Q PCR 316 system can achieve rapid thermal cycling by circulating pre-heated liquids in microfluidic chips positioned over the heaters, enabling the performance of 30 cycles of real-time PCR in 15 minutes [15, 16]. Our findings were also consistent with previous findings that PCR processing time was significantly reduced to $<40$ minutes (for 45 cycles) with the Veri-Q PCR 316 system compared with other real-time PCR systems, which took approximately two hours for amplification [17].

In conclusion, we demonstrate that both the SFTS-QS and
PowerChek kits yielded accurate results, but the SFTS-QS kit required a shorter total turnaround time. Based on the high speed and simplicity, the SFTS-QS kit is a potentially useful diagnostic tool for the rapid detection of SFTSV.

\section{AUTHOR CONTRIBUTIONS}

IY Yoo and HJ Huh designed the study, analyzed the data, and wrote the manuscript. YK Yoon, HJ Huh, and JY Kim collected the samples. JY Kim participated in experiments. HJ Huh and NY Lee reviewed the manuscript. All authors read and approved the final manuscript.

\section{CONFLICTS OF INTEREST}

No potential conflicts of interest relevant to this paper were reported.

\section{RESEARCH FUNDING}

This research was supported by a grant from the Korea Health Technology R\&B Project through the Korea Health Industry Development Institute (KHIDI), funded by the Ministry of Health \& Welfare, Korea (grant No.: HI16C1048).

\section{ORCID}

In Young Yoo

Ji-Youn Kim

Young Kyung Yoon

Hee Jae Huh

Nam Yong Lee
https://orcid.org/0000-0003-1505-846X https://orcid.org/0000-0002-9206-7338 https://orcid.org/0000-0001-8435-935X https://orcid.org/0000-0001-8999-7561 https://orcid.org/0000-0003-3688-0145 


\section{REFERENCES}

1. Yu XJ, Liang MF, Zhang SY, Liu Y, Li JD, Sun YL, et al. Fever with thrombocytopenia associated with a novel bunyavirus in China. N Engl J Med 2011;364:1523-32.

2. Liu Q, He B, Huang SY, Wei F, Zhu XQ. Severe fever with thrombocytopenia syndrome, an emerging tick-borne zoonosis. Lancet Infect Dis 2014; 14:763-72.

3. Guo CT, Lu QB, Ding SJ, Hu CY, Hu JG, Wo Y, et al. Epidemiological and clinical characteristics of severe fever with thrombocytopenia syndrome (SFTS) in China: an integrated data analysis. Epidemiol Infect 2016;144:1345-54.

4. Wen HL, Zhao L, Zhai S, Chi Y, Cui F, Wang D, et al. Severe fever with thrombocytopenia syndrome, Shandong Province, China, 2011. Emerg Infect Dis 2014;20:1-5.

5. Xu H, Zhang L, Shen G, Feng C, Wang X, Yan J, et al. Establishment of a novel one-step reverse transcription loop-mediated isothermal amplification assay for rapid identification of RNA from the severe fever with thrombocytopenia syndrome virus. J Virol Methods 2013;194:21-5.

6. Cui L, Ge Y, Qi X, Xu G, Li H, Zhao K, et al. Detection of severe fever with thrombocytopenia syndrome virus by reverse transcription-crosspriming amplification coupled with vertical flow visualization. J Clin Microbiol 2012;50:3881-5.

7. Huang XY, Hu XN, Ma H, Du YH, Ma HX, Kang K, et al. Detection of new bunyavirus RNA by reverse transcription-loop-mediated isothermal amplification. J Clin Microbiol 2014;52:531-5.

8. Kim JY, Koo B, Jin CE, Kim MC, Chong YP, Lee SO, et al. Rapid diagnosis of tick-borne illnesses by use of one-step isothermal nucleic acid amplification and bio-optical sensor detection. Clin Chem 2018;64:55665.
9. Sun Y, Liang M, Qu J, Jin C, Zhang Q, Li J, et al. Early diagnosis of nove SFTS bunyavirus infection by quantitative real-time RT-PCR assay. J Clin Virol 2012;53:48-53.

10. Yoshikawa T, Fukushi S, Tani H, Fukuma A, Taniguchi S, Toda S, et al. Sensitive and specific PCR systems for detection of both Chinese and Japanese severe fever with thrombocytopenia syndrome virus strains and prediction of patient survival based on viral load. J Clin Microbiol 2014;52:3325-33.

11. Lee SH, Kim HJ, Byun JW, Lee MJ, Kim NH, Kim DH, et al. Molecular detection and phylogenetic analysis of severe fever with thrombocytopenia syndrome virus in shelter dogs and cats in the Republic of Korea. Ticks Tick Borne Dis 2017;8:626-30.

12. Yun SM, Lee WG, Ryou J, Yang SC, Park SW, Roh JY, et al. Severe fever with thrombocytopenia syndrome virus in ticks collected from humans, South Korea, 2013. Emerg Infect Dis 2014;20:1358-61.

13. CLSI. Evaluation of detection capability for clinical laboratory measurement procedures. 2nd ed. CLSI EP17-A2. Wayne, PA: Clinical and Laboratory Standards Institute. 2012.

14. CLSI. User verification of precision and estimation of bias. 3rd ed. CLSI EP15-A3. Wayne, PA: Clinical and Laboratory Standards Institute. 2014.

15. Neuži P, Giselbrecht S, Länge K, Huang TJ, Manz A. Revisiting lab-ona-chip technology for drug discovery. Nat Rev Drug Discov 2012;11: 620-32.

16. Houssin T, Cramer J, Grojsman R, Bellahsene L, Colas G, Moulet H, et al. Ultrafast, sensitive and large-volume on-chip real-time PCR for the molecular diagnosis of bacterial and viral infections. Lab Chip 2016;16: 1401-11.

17. Lee SH, Kim SW, Lee S, Kim E, Kim DJ, Park S, et al. Rapid detection of Mycobacterium tuberculosis using a novel ultrafast chip-type real-time polymerase chain reaction system. Chest 2014;146:1319-26. 